\title{
JUVENILE IDIOPATHIC ARTHRITIS - A CASE REPORT
}

Paresh H. Bharia, S. D. Birare, S. S. Dantkale, S. A. Chaware, V. B. Dhurve

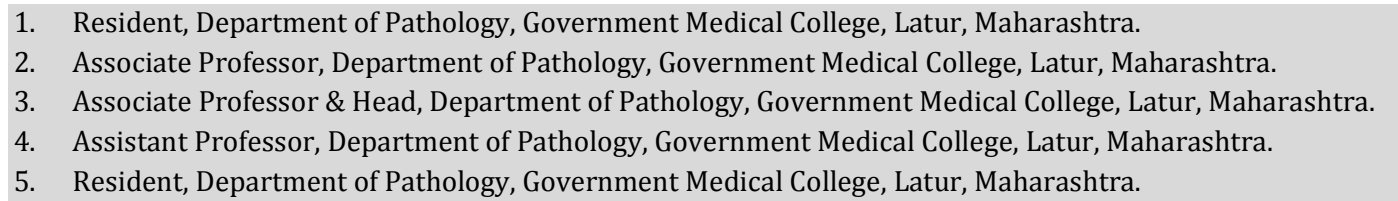

\section{CORRESPONDING AUTHOR}

Paresh H. Bharia,

Department of Pathology,

Government Medical College, Latur,

E-mail: bharia.paresh@gmail.com,

Ph: 00919975188055.

ABSTRACT: The prevalence of Juvenile idiopathic arthritis (JIA) is 0.86 per 1000 children. Subcutaneous nodules have been reported in $5 \%$ to $10 \%$ of children with JIA. Approximately $90 \%$ of patients with $\mathrm{RA}$ and subcutaneous nodules test positive for rheumatoid factor (RF), and approximately $40 \%$ of all RF-seropositive patients with RA have subcutaneous nodules, whereas only $6 \%$ involvement is seen in seronegative cases. We hereby report a case of atypical Juvenile idiopathic arthritis (JIA) in a 6 year old, female child with joint pain \& myalgia along with subcutaneous nodules over the dorsum of feet, hands and elbows. Joint pain initially involving the left ankle, slowly progressed to involve the knee, shoulder, wrist, metacarpophalangeal and interphalangeal joints over a period of one year. Joint involvement was not symmetric. RF was Negative. Fundoscopy examination was normal. Histopathological examination revealed a central zone of Fibrinoid necrosis surrounded by epithelioid histiocytes and occasional lymphocytes. Differential diagnosis of Rheumatoid Nodule (RN) or Subcutaneous Granuloma Annulare (SGA) or Necrobiosis Lipoidica Diabeticorum was made. In light of clinicopathological findings, both SGA and NLD were ruled out and the diagnosis of Juvenile idiopathic arthritis presenting as RF-negative polyarthritis was made.

KEYWORDS: juvenile idiopathic arthritis, rheumatoid arthritis, rheumatoid nodule.

MESH TERMS: Nil

INTRODUCTION: Juvenile idiopathic arthritis (JIA), previously known as juvenile rheumatoid arthritis encompasses all forms of arthritis that develop before 16 years of age and persist for a minimum of 6 weeks. Rheumatoid nodules (RN) are the most common cutaneous lesion in adult cases of rheumatoid arthritis (RA) and are present in $25 \%$ cases (both oligoarticular \& polyarticular) [1]. A much greater incidence $(75 \%)$ is observed in those with RA-associated Felty syndrome. [2] Approximately 90\% of patients with RA and subcutaneous nodules test positive for rheumatoid factor (RF), and approximately $40 \%$ of all RF-seropositive patients with RA have subcutaneous nodules, whereas only $6 \%$ involvement is seen in seronegative cases. [3] Rheumatoid nodules are clinical predictors of more severe arthritis, seropositivity, joint erosions, and rheumatoid vasculitis. It has been suggested that the presence of RNs often requires more aggressive treatment of the underlying RA to prevent sequelae. However, in some cases disease 
progression is independent of nodular disease activity. The prevalence of JIA is 0.86 per 1000 children. Subcutaneous nodules have been reported in $5 \%$ to $10 \%$ of children with JIA. [4] Rheumatoid nodules are almost always confined to those children with polyarticular arthritis i.e. involving $\geq 5$ joints in first 6 months. [5] Genetics seems to play a role in the appearance of RNs. The HLA-DR4 haplotype (including the heterogeneous group of DRB1 alleles) is predictive of the risk of subcutaneous nodules in RA. [6]

CASE HISTORY: We hereby report a case of an atypical Juvenile idiopathic arthritis in a 6 year old, female child with joint pain \& myalgia. Joint pain initially involved the left ankle, slowly progressing to involve the knee, shoulder, wrist, metacarpophalangeal and interphalangeal joints over a period of one year. Joint involvement was not symmetric. There was presence of subcutaneous nodules over the dorsum of feet [Figure 1], hands and elbows. Clinical diagnosis of Rheumatoid arthritis was made.

On investigating, rheumatoid factor was negative. Serum ANA and anti-CCP (cyclic citrullinated peptide) antibody tests were advised but not performed. Fundoscopy examination was normal. A skin biopsy was taken from the site of the subcutaneous nodule from the dorsum of the feet. Histopathological examination of the subcutaneous nodule revealed a central zone of fibrinoid necrosis surrounded by epithelioid histiocytes and occasional lymphocytes. [Figures 2 \& 3] Differential diagnosis of Rheumatoid Nodule or Subcutaneous Granuloma Annulare (SGA) or Necrobiosis Lipoidica Diabeticorum (NLD) was made.

DISCUSSION: A diagnosis of rheumatoid nodules is made in the clinical context of the disease. Although biopsies of subcutaneous nodules are occasionally done, they are not useful for diagnosis. [7] The 2010 ACR-EULAR criteria of rheumatoid arthritis has not taken into consideration the presence of rheumatoid nodules or radiographic joint damage because these findings occur rarely in early RA. [8] Many different types of subcutaneous nodules are histologically identical to rheumatoid nodules; mainly NLD and SGA, which are often misdiagnosed as RNs. [9]

In general, RNs tend to be larger and to be confined to the deep dermis or subcutis, at the bony prominences, commonly located at the ulnar aspect of the forearm, elbows, occiput, and lumbosacral area. In our case, the nodules were present on bony prominences of the dorsum of feet and hands. Microscopically they have a central zone of fibrinoid necrosis surrounded by a prominent rim of epithelioid histiocytes and numerous lymphocytes and plasma cells. [1] Fibrin may be deposited in the centre of the granulomas, as opposed to the mucin deposition of SGA. [10] However, RNs with abundant mucin may be present. [11] Therefore histopathological differentiation between RNs and SGA is difficult.

Serum IgM RF has been found in $75-80 \%$ of patients with RA [8]; therefore, a negative result does not exclude the presence of this RA in our patient.

Subcutaneous granuloma annulare (SGA) is one of the most common dermatoses with the involvement of skin and/or subcutis, usually seen in adults and children, but the aetiology and pathogenesis are unclear. [12] It most often manifests as a large, asymptomatic soft tissue mass. Although nodules are usually stable for months, they may rapidly enlarge over the course of weeks. The typical lesions of SGA are single or multiple, small, pinkish, nonulcerated nodules in the deep subcutaneous tissue. The most 
common lesion location is lower extremity, especially the peri-tibial area, followed by the hands. On histological evaluation, these nodules are similar to the nodules seen in adults with rheumatoid arthritis and to the lesion recognized in adult diabetic patients as necrobiosis lipoidica diabeticorum. [13] Characteristically, the necrobiotic focus is not so large or deeply situated as those of rheumatoid nodules or as broad and diffuse as those of necrobiosis lipoidica (diabeticorum). [9] In our case, one year history of joint pain and migratory polyarthritis along with subcutaneous nodules ruled out SGA and NLD.

Necrobiosis Lipoidica is a well-recognized dermatologic complication of diabetes mellitus and may develop in both juvenile (Type I) and adult-onset (Type II) diabetes mellitus. Positive family history of diabetes mellitus has also been one of the risk factors. [13] The sclerodermatous plaque is round to oval, red-brown with an elevated rim. Like SGA, the peri-tibial region is the most common site. Clinical correlation must be taken into consideration before making the diagnosis of NLD. [11] Our patient was not diabetic nor gave any positive family history for diabetes mellitus. The patient was successfully treated with steroids and has not shown sustained disease or diabetes mellitus over a one year follow up.

In light of clinicopathological findings, the diagnosis of Juvenile idiopathic arthritis presenting as RF-negative polyarthritis was made. We thus believe that histopathology of rheumatoid nodule presents a special challenge to a pathologist. Relevant clinicopathological details are essential for diagnosis and appropriate management.

\section{REFERENCES:}

1. Kumar V, Abbas A K, Fausto N, Aster J C. Robbins \& Cotran Pathologic Basis of Disease. 8 th Edition. Philadelphia: Saunders Elsevier; 2010.

2. Sayah A, English JC 3rd: Rheumatoid arthritis: A review of the cutaneous manifestations. J Am Acad Dermatol 53:91-209, 2005

3. Kaye BR, Kaye RL, Bobrove A: Rheumatoid nodules. Review of the spectrum of associated conditions and proposal of a new classification, with a report of four seronegative cases. Am J Med 76:279-292, 1984

4. Vicente García-Patos. Rheumatoid Nodule. Semin Cutan Med Surg 26:100107 (C) 2007 Elsevier Inc

5. Sampath Prahalad and David $\mathrm{N}$ Glass. Is juvenile rheumatoid arthritis/juvenile idiopathic arthritis different from rheumatoid arthritis?

6. Ahmed SS, Arnett FC, Smith CA, et al: The HLA-DRB1*0401 allele and the development of methotrexate-induced accelerated rheumatoid nodulosis: A follow-up study of 79 Caucasian patients with rheumatoid arthritis. Medicine 80:271-278, 2001

7. Patricia T. Ting and Benjamin Barankin. Dermacase. VOL 5. JANUARY 2005 Canadian Family Physician.

8. Longo DL, Fauci AS, Kasper DL et al. Harrison's Principles of Internal Medicine. Volume 2. 18th edition, New York. McGraw Hill Publications. 2012.

9. Hakan Sabuncuoglu et al. Subcutaneous Granuloma Annulare of the Scalp in Childhood: A Case Report and Review of the Literature. Turkish Neurosurgery 2007, Vol: 17, No: 1, 19-22

10. Mills SE, Carter D, Greenson JK et al. Sternberg's Diagnostic surgical pathology. 5 th Edition. Philadelphia. Lippicott Williams \& Wilkins. 2010. 
11. Mckee P H, Calonje E, Granter S R. Pathology of the skin with clinical correlations. Volume 1. 3 rd edition. Philadelphia. Elsevier Mosby. 2005.

12. Requena L, Fernandez- Figueras MT. Subcutaneous granuloma annulare. Semin Cutan Med Surg 2007; 26: 96-9

13. Grogg KL, Nascimento AG. Subcutaneous granuloma annulare in childhood: clinicopathologic features in 34 cases. Pediatrics: 107(3):42, 2001

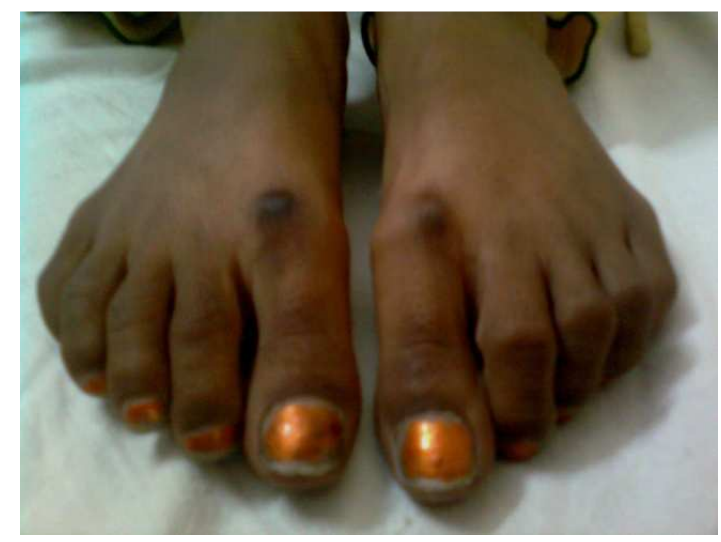

Figure 1. Subcutaneous nodules present on the dorsum of foot.

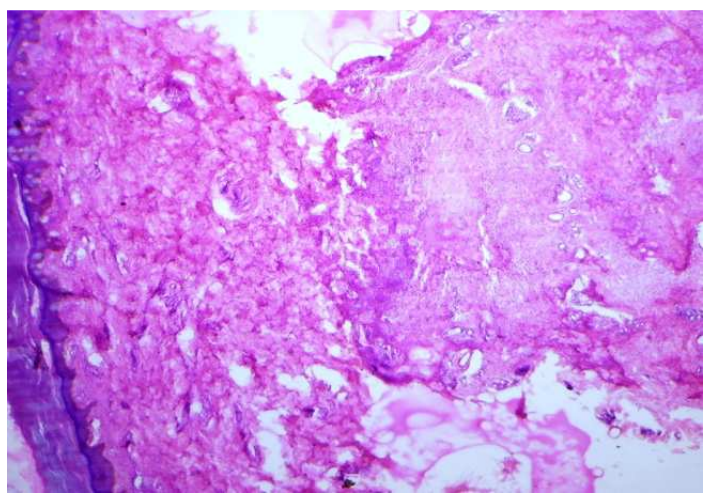

Figure 2. Low power view through subcutaneous nodule showing a necrobiotic focus situated in the deeper dermis. (10x; H\&E)

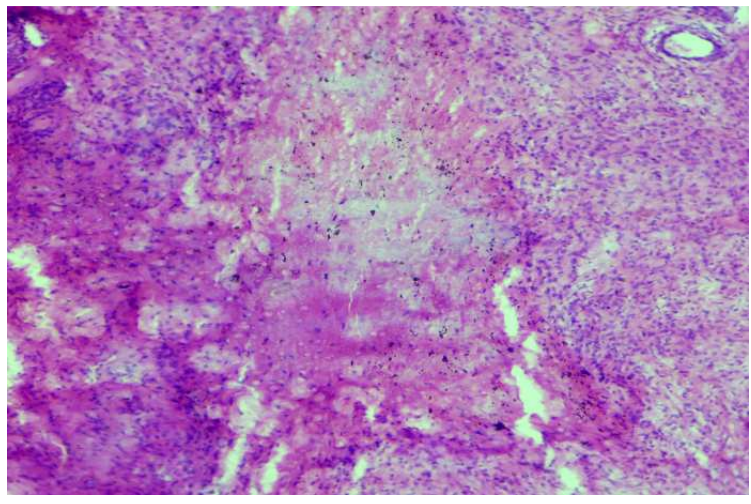

Figure 3. Necrobiotic focus showing central fibrinoid necrosis surrounded by histiocytes and lymphocytes (40x; H\&E) 